\title{
Carcass composition of jungle fowl in comparison with broilers and indigenous chicken.
}

\begin{abstract}
Carcass composition of three breed of chicken was compared: jungle fowl, broiler and Malaysian indigenous chicken. The chickens were sacrificed and were divided into forequarter and hindquarter. The forequarter was further divide into breast, wing and ribs. The muscle, bone, fat and skin of all different portions were separated, weighed and recorded. The results showed that broilers have significantly higher muscle weight compared to indigenous chicken and jungle fowl. The jungle fowl has significantly higher bone weight with least fat compared to the other two breeds The carcass composition of indigenous chicken is always in between the broiler and jungle fowl. Present results show that different habitat and feeding pattern of these chickens do contribute to these changes.
\end{abstract}

Keyword: Broiler; Indigenous chicken; Jungle fowl; Meat composition. 